\title{
Internally and externally induced deformations of the magnetospheric equatorial current as inferred from spacecraft data
}

\author{
N. A. Tsyganenko, V. A. Andreeva, and E. I. Gordeev \\ Institute and Faculty of Physics, Saint Petersburg State University, Saint Petersburg, Russia \\ Correspondence to: N. A. Tsyganenko (nikolai.tsyganenko@gmail.com)
}

Received: 11 July 2014 - Revised: 23 October 2014 - Accepted: 28 November 2014 - Published: 6 January 2015

\begin{abstract}
Based on a data pool of 79 yearly files of space magnetometer data by Polar, Cluster, Geotail, and THEMIS satellites between 1995 and 2013, we developed a new quantitative model of the global shape of the magnetospheric equatorial current sheet as a function of the Earth's dipole tilt angle, solar wind ram pressure, and interplanetary magnetic field (IMF). This work upgrades and generalizes an earlier model of Tsyganenko and Fairfield (2004) by extending the modeling region to all local times, including the dayside sector. In particular, an essential feature of the new model is the bowl-shaped tilt-related deformation of the equatorial surface of minimum magnetic field, similar to that observed at Saturn, whose existence in the Earth's magnetosphere has been demonstrated in our recent work (Tsyganenko and Andreeva, 2014).
\end{abstract}

Keywords. Magnetospheric physics (magnetospheric configuration and dynamics)

\section{Introduction}

The global geometry of the Earth's distant magnetic field is determined by the position and shape of principal boundaries or sheets, where most of the magnetospheric electric currents are concentrated. Of foremost importance is the magnetopause, carrying the Chapman-Ferraro current and containing the magnetic flux of terrestrial origin. The other essential region is the equatorial current sheet, forming the magnetotail structure on the nightside and partially extending to the dayside in the form of the ring current. The large-scale configuration and magnitude of those currents is controlled by the external factors such as the solar wind ram pressure and the interplanetary magnetic field (IMF). Owing to the tilted orientation of the Earth's dipole axis with respect to the di- rection of the solar wind flow, the magnetospheric equatorial currents exhibit basic asymmetries, whose modeling was a subject of numerous works in the past. To our knowledge, the most recent and detailed study in that area was done by Tsyganenko and Fairfield (2004; referred to henceforth as TF04), who developed a detailed empirical model of the tiltand IMF-related asymmetries in the observed shape of the tail current sheet, using magnetometer data of Geotail and Polar spacecraft taken during 1994-2002 and 1998-2002, respectively. The goal of the present work is to revise and extend the TF04 model using a much larger data set and taking into account new results concerning the global deformation of the near-equatorial magnetic field configuration due to the seasonal/diurnal variations of the geodipole tilt angle. Namely, as was shown in our recent work (Tsyganenko and Andreeva, 2014; referred to henceforth as TA14), in a simple vacuum magnetosphere with a shielded and tilted planetary dipole, the surface of normalized minimal magnetic mirror ratio $B_{\min } / B$ takes the form of an asymmetric bowl, notably resembling in its shape the bowl-shaped current disk discovered in Saturn's magnetosphere (Arridge et al., 2008). Because the electric currents in equilibrium configurations tend to concentrate in the regions with the weakest magnetic field, we concluded that the bowl-shaped deformation is a natural result of the combination of two basic types of magnetospheric asymmetries: the day-night asymmetry due to the solar wind compression and the north-south asymmetry due to the planetary dipole tilt. Based on this conjecture and using a large set of data from the Polar, Cluster, Geotail, and THEMIS satellites, we found that the bowl-like deformation is indeed clearly present in the terrestrial magnetosphere and reveals itself in the shape of a best-fit surface, approximating the location of the near-equatorial region where the radial component of the magnetic field changes polarity. 
In the present paper we extend the TA14 study and derive an analytic model of the shape of the equatorial $\min |\boldsymbol{B}|$ surface (termed TAG14 for future reference), with the deformation parameters quantified as functions of the solar wind ram pressure and IMF components. The following Sect. 2 describes our data set and the selection criteria used to generate a subset of the current sheet crossings. Section 3 outlines the mathematical model representing the shape of the current sheet and the numerical procedure to derive the model parameters. In Sect. 4 we provide details of the model parameterization by the interplanetary input data and describe the model optimization method. Section 5 presents main results of fitting the model to the data, graphically illustrates the effects of the controlling variables on the shape of the equatorial current, and compares the newly developed model with the TF04. Section 6 discusses limitations of the model and unaccounted factors that may affect the equatorial current geometry, in particular, a possible effect of the IMF $B_{x}$ component on the position of the tail current sheet detected in magnetohydrodynamic (MHD) simulations. Section 7 concludes the paper.

\section{Data}

A principal factor in the data-based modeling is the range and evenness of the data coverage in both geometrical and parametrical space. Since the publication of the TF04 model based on Geotail and Polar magnetometer observations, a huge volume of new data has become available, owing first of all to multi-satellite long-term missions such as THEMIS and Cluster. In addition, new data from Geotail and Polar taken during 2002-2012 and 2002-2008, respectively, have been acquired. This data offered an attractive opportunity to integrate a large amount of new experimental information into the new model. In the following, we describe the general procedures and then provide more specific details on the data from each mission.

The basic operations involved in the data processing included the following. The original data records (typically, with 1 min resolution) with average position and magnetic field vectors were first merged with available OMNI interplanetary data (time shifted to the standard subsolar bowshock location; see King and Papitashvili, 2005). Then the consolidated data files were manually edited, one day worth of data at a time, to filter out magnetosheath and solar wind intervals. The visual selection of magnetospheric data was aided by corresponding plots of the expected magnetopause position, calculated from the boundary model of Lin et al. (2010) driven by concurrent values of the solar wind ram pressure and IMF $B_{z}$. The edited magnetospheric data were averaged over 5 min intervals and each output data record was tagged with all relevant OMNI information, including not only the current time moment but also a 35 min trail of preceding data, to take into account time lags due to the de- layed reaction of the magnetosphere to the external input. Owing to availability of data on the solar wind $V_{y}$ and $V_{z}$ velocity components, all vectors were transformed to the geocentric solar wind (GSW) coordinate system with the $X_{\mathrm{GSW}}$ axis directed anti-parallel to the observed solar wind flow vector (Hones et al., 1986; Tsyganenko et al., 1998; TF04). The final result of these procedures was a set of 79 yearly data files, containing the edited magnetospheric data taken by each of the individual satellites, briefly described below.

\subsection{Geotail}

Magnetic field data of Geotail included 18 yearly files, partly inherited from the old set (1995-2002) used in TF04, and complemented by newer data for 2002-2012. Due to the relatively high perigee of Geotail $\left(\sim 10 R_{\mathrm{E}}\right)$, its data did not cover the inner and dayside magnetosphere and served mostly as the main source of information on the mid-tail field configuration. All the data were corrected for the small $B_{z}$ offset as described in detail in an earlier publication (Tsyganenko, 2002), using the daily offset tables from the Data ARchives and Transmission System (DARTS) website: http://darts.isas.jaxa.jp/stp/geotail/bzoffset.html. A comprehensive description of the mission and its magnetic field experiment was given by Nishida (1994) and Kokubun et al. (1994). The total number of the Geotail 5 min data records in the 18 final subsets was 575981 , equivalent to $\sim 5.5$ years worth of continuous observations.

\subsection{Polar}

The entire duration of the Polar mission was about 12 years between March 1996 and April 2008. The magnetic field (MGF) experiment onboard Polar was described by Russell et al. (1995). The original 1 min average magnetic field data were downloaded from the UCLA Polar website (http:// www-ssc.igpp.ucla.edu/forms/polar/plot_low.html) and then processed as outlined in the beginning of this section. Due to the gradual precession of Polar's line of apsides, the spacecraft apogees occurred in the near-equatorial magnetosphere during only a relatively limited time interval between 2001 and 2004. Moreover, during the solstice periods with the largest tilt angles (hence, with the largest current sheet deformation), the apogees of Polar were located in the dawn-dusk sector, which also limited their value as a source of information on the tilt-related warping of the equatorial current in the noon-midnight plane. On a positive side, due to its relatively low apogee and its short orbital period, Polar provided a decent coverage of the inner magnetosphere, with the total of 891260 data records in the final subset, i.e., about $\sim 8.5$ years of continuous magnetospheric observations.

\subsection{Cluster}

Magnetometer observations from Cluster mission used in this study spanned the period from February 2001 through 
April 2013 and comprised 5 min average vectors taken by the Cluster 3 probe. The tetrahedron orbital configuration of the Cluster satellites was specially designed for multipoint simultaneous observations of small-scale magnetospheric structures and dynamic phenomena, which greatly enhanced the scientific return of the mission. However, since our study is focused on the large-scale statistically averaged configuration of the equatorial current, it would not make much sense to use the data of all four probes due to their proximity to each other (and, hence, close coherence between their observations), and that was the reason behind using the data of only one Cluster probe.

The original $1 \mathrm{~min}$ resolution data were downloaded from the NSSDC CDAWEB (Coordinated Data Analysis Web) site and then underwent the above-described filtering/editing procedure. The total number of Cluster $5 \mathrm{~min}$ data records selected into the final yearly files was 514112 , equivalent to $\approx 4.9$ years worth of observations in the magnetosphere. More details on the Cluster FGM experiment can be found in Balogh et al. (1997).

\subsection{THEMIS}

THEMIS data played a special role in our study owing to their excellent coverage of all longitude sectors around solstice seasons, which in particular made it possible to reveal the details of the magnetic deformation on the dayside, the region mostly uncharted by Geotail and insufficiently covered by Polar data. The data of five THEMIS probes covered the time interval from their launch at the end of February 2007 through July 2013. The data processing procedures were essentially the same as those for the other spacecraft. Most of the data were provided by the relatively low-apogee THEMIS A-, D-, and E-probes: 433 847, 431 337, and 442914 records, respectively, which is equivalent to a total of $\sim 12.4$ years worth of observation time of all three probes. The high-apogee B- and C-probes yielded much less data, respectively, 137561 and 189617 5 min records (a total of $\sim 3.1$ years), which is comparable to that of Geotail. For a comprehensive information on THEMIS mission, spacecraft orbits, and magnetometer experiment, the reader is referred to Angelopoulos (2008) and Auster et al. (2008).

\subsection{Compilation of the crossings data set}

The next procedure was to compile a single subset of data taken in close vicinity of the equatorial current, to be used as input by the model fitting algorithm. To that end, all the 79 yearly files were first subject to additional filtering to exclude cases of abnormal magnetospheric or interplanetary conditions, in particular, storm-time periods. More specifically, the filtering criteria were such that only records with the IMF magnitude $B<10 \mathrm{nT}$, solar wind ram pressure $0.1 \leq P_{\mathrm{dyn}} \leq 10 \mathrm{nPa}$, and the activity index $-100 \leq \mathrm{SYM}$ -

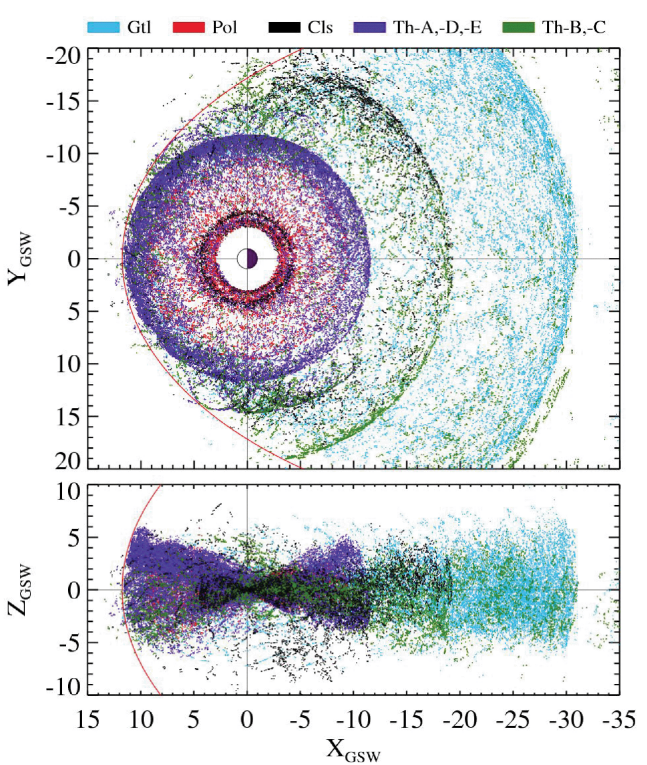

Figure 1. Distribution of data points in the crossings set, projected onto GSW equatorial (top) and noon-midnight (bottom) planes.

$\mathrm{H} \leq 30 \mathrm{nT}$ were retained. In addition, for each data record a corresponding estimate of the warped model neutral sheet position $Z_{\mathrm{N}}=Z_{\mathrm{N}}\left(x, y, \Psi, P_{\mathrm{dyn}}, B_{z}^{(\mathrm{IMF})}\right)$ was calculated in the GSW coordinates, based on the TF04 model (smoothly extrapolated to the dayside whenever necessary), and all points with $\left|z-Z_{\mathrm{N}}\right|>4 R_{\mathrm{E}}$ were also removed, to exclude higher-latitude tail lobe data irrelevant for our study. Finally, we restricted the tailward extension of the data by setting the limit on $X_{\mathrm{GSW}} \geq-60 R_{\mathrm{E}}$. That resulted in a further reduction of the data set volume.

After having stored the selected records into the memory, the code proceeded along the entire array and identified those records for which at least one immediate neighbor (i.e., either the preceding or next 5 min average) had the opposite polarity of the magnetic field radial component $B_{\mathrm{r}}$. As expected, only a relatively small fraction of the data (in total, 109363 records) satisfied that condition and were included in the output crossings file. A more detailed discussion of the adopted selection criterion will be presented in Sect. 6; here we only briefly note that it can be justified in at least two ways. First, the reversals of $B_{\mathrm{r}}$ usually occur when a spacecraft enters the region of the weakest $B$ magnitude near the field line apex, co-located with areas of the greatest plasma pressure and electric current. Second, multiple $B_{\mathrm{r}}$ reversals typically occur in the regions with high levels of magnetic field noise and large plasma beta, also a common feature of the magnetospheric equatorial current.

Figure 1 illustrates the spatial distribution of the crossings file data, with contributions from individual spacecraft shown by different colors. The multiple peaks of the data density correspond to the satellite apogees, where their orbital speed was the lowest. As can be seen from the plots, the coverage 

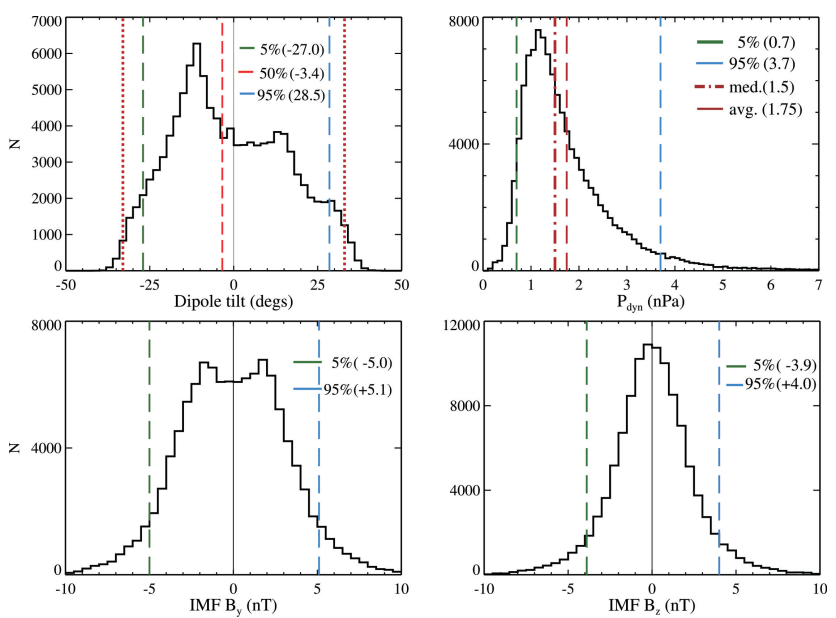

Figure 2. Histograms of the dipole tilt angle $\Psi$ (top left), solar wind ram pressure $P_{\text {dyn }}$ (top right), IMF $B_{y}$ (bottom left), and IMF $B_{z}$ (bottom right), corresponding to the subset of equatorial current sheet crossings shown in Fig. 1. The colored dashed lines show the $5 \%$, median, average, and $95 \%$ values as explained in legends. Red dotted lines in the $\Psi$ histogram show the limiting values $\pm 33^{\circ}$, corresponding to the standard geocentric solar magnetospheric (GSM) coordinate system (see text for details).

is fairly dense and sufficiently uniform in local time without outstanding gaps.

Our goal is to derive from data not only the average geometry of the tilt-related deformation of the equatorial current but also its response to changing interplanetary conditions. The amount of information in this regard and, hence, the range of validity of the model critically depends on the overall span and relative density of the data distribution in the parametric space $\left\{\Psi, P_{\mathrm{dyn}}, B_{y}^{(\mathrm{IMF})}, B_{z}^{(\mathrm{IMF})}\right\}$. Figure 2 illustrates the coverage in the form of histograms of these four quantities, based on 109363 records in the compiled crossings data set. The dipole tilt angle histogram is notably asymmetric with a clear shift towards negative values: about $\sim 56 \%$ of records have $\Psi<0$ and the median value of the distribution equals $-3.35^{\circ}$. The asymmetry, however, is not a factor here, because the tilt-related deformations must be symmetric with respect to the geocentric solar magnetospheric (GSM) equatorial plane, and these symmetry properties are explicitly built in the model by construction. One more feature to be noted is a relatively small part of the tilt angle distribution in Fig. 2a that spills beyond the limits $\Psi= \pm 33^{\circ}$ (red dotted lines), corresponding to the standard GSM coordinate system. In this work (as in TF04), we use the GSW system which takes into account the actual direction of the solar wind flow, and the data that fall outside the standard limits are those taken around solstices, at times of a significantly non-radial solar wind.

The histograms of interplanetary quantities are in general similar to those presented in TF04 (cf. their Fig. 1), although the overall range and all characteristic values of both the ram
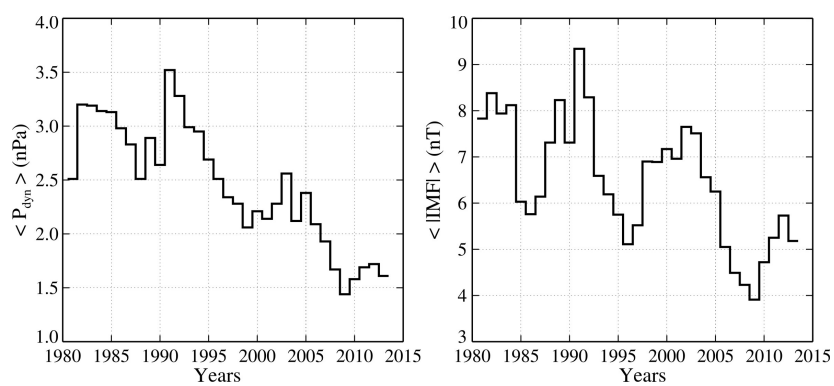

Figure 3. Variation of the annual averages of the solar wind ram pressure (left) and of the IMF magnitude (right) for the period from 1981 through 2013. A long-term decreasing trend for both quantities is clearly seen.

pressure and the IMF are significantly lower in the present case: the median value of $P_{\text {dyn }}$ went down to 1.5 from 2.0, while the 5-95\% ranges of IMF $B_{y}$ and $B_{z}$ shrank from \pm 7 to \pm 5 and from \pm 5 to $\pm 4 \mathrm{nT}$, respectively. The most likely reason is that about two-thirds of our data in the crossings file fall on the period 2007-2012 with very low solar activity and weaker solar wind pressure, while the TF04 data set covered mostly earlier years with a larger average $\left\langle P_{\text {dyn }}\right\rangle$. This conjecture is confirmed by Fig. 3 with plots of annual averages of the wind ram pressure and the IMF magnitude for the period 1981-2013, calculated on the basis of yearly OMNI data with 5 min resolution. Both parameters display not only periodic solar cycle variations but also a clear descending trend between the last three peaks in 1991, 2002-2003, and 2012.

\section{Mathematical model of the field reversal surface}

In formulating the model, we followed the earlier employed approach, that is, start from the simplest form satisfying the most obvious and essential a priori requirements, perform trial fitting runs, and then make the model more flexible by adding first-order correction terms or factors. In the lowest approximation, it is natural to describe the bowlshaped configuration with an axially symmetric surface $z=$ $Z_{\mathrm{s}}(\rho, \Psi)$ in the solar-magnetic cylindrical coordinate system $\{\rho, \phi, z\}$, with $\rho=\sqrt{x^{2}+y^{2}}$ and the $z$ axis anti-parallel to the geodipole magnetic moment and tilted by the angle $\Psi$ to the terminator plane. Near a planet, the surface is smoothly tangent to the dipole equatorial plane, while at large distances, $\rho \rightarrow \infty$, the slope $\partial Z_{\mathrm{s}} / \partial \rho$ must tend to $-\tan \Psi$, in order that the bowl generatrix line becomes asymptotically parallel to the solar wind flow in the distant magnetotail. A very simple equation, perfectly satisfying these requirements was suggested in TA14 as

$Z_{\mathrm{s}}=R_{\mathrm{H}} \tan \Psi\left\{1-\left[1+\left(\frac{\rho}{R_{\mathrm{H}}}\right)^{\alpha}\right]^{1 / \alpha}\right\}$, 
where $R_{\mathrm{H}}$ is the current sheet hinging distance and the $\alpha$ parameter controls the curvature of the current sheet in the transition region $\rho \sim R_{\mathrm{H}}$ between the inner and outer magnetosphere. A rough theoretical estimate (Tsyganenko, 1998; Sect. 3) yields $\alpha \sim 4$; in the present work, as in TF04 and TA14, we treated $\alpha$ as a variable unknown parameter to be derived from the data. Here we note in passing that the TF04 model current sheet was formulated somewhat differently: that model was intended only for the night sector, and for that reason the current sheet was represented from the outset by a warped trough-like surface in the solar-magnetospheric (SM) or solar-wind (SW) coordinates, while in this work we describe the sheet in the SM system.

Now we note that Eq. (1) corresponds to a strictly axisymmetric surface in the SM coordinates, which naturally prompts us to add more degrees of freedom and generalize the model by allowing the distance $Z_{\mathrm{s}}$ between the sheet and equatorial plane to vary with longitude $\phi$. The modified equation of the sheet surface takes the form

$$
\begin{aligned}
Z_{\mathrm{s}} & =R_{\mathrm{H}} \tan \Psi\left\{1-\left[1+\left(\frac{\rho}{R_{\mathrm{H}}}\right)^{\alpha}\right]^{1 / \alpha}\right\} \\
& \times\left(a_{0}+a_{1} \cos \phi+a_{2} \sin \phi\right),
\end{aligned}
$$

where the a priori unknown Fourier coefficients $a_{1}$ and $a_{2}$ quantify the degree of possible day-night and dawn-dusk asymmetry of the bowl. As discussed in TA14, the most interesting parameter here is the first coefficient $a_{1}$ : its negative best-fit value would indicate a lesser degree of warping on the dayside, or (with $a_{1}=-a_{0}$ ) even no warping at all in the noon sector. Conversely, a positive value would manifest a stronger warping. Our most important finding in TA14 was that the coefficient $a_{1}$ is large and positive, which indicated a significantly enhanced anti-sunward deflection of the dayside brim of the bowl away from the SM equator. It was also found (and further confirmed in this study) that the dawndusk asymmetry term $a_{2} \sin \phi$ is always negligibly small in comparison with the first two terms in Eq. (2) and, for that reason, it was discarded.

The next step is to further generalize the model by taking into account principal effects that are associated with the state of the interplanetary medium. They can be divided into two categories: (a) effects of the solar wind ram pressure $P_{\mathrm{dyn}}$ and of the IMF components that affect the tilt-dependent deformation via the parameters in the model Eq. (2), and (b) effects unrelated to the dipole tilt, i.e., deformations that show up even at $\Psi=0$ and, hence, should be introduced by adding separate terms in Eq. (2). The effects of the category (a) can be modeled by representing the parameters $R_{\mathrm{H}}, \alpha, a_{0}$, and $a_{1}$ in Eq. (2) as appropriate functions of $P_{\text {dyn }}$ and IMF $B_{z}$; their specific forms will be addressed in more detail below. The most significant effect of category (b) is the gradual twisting of the tail current sheet under the influence of the azimuthal component $B_{y}$ of the IMF (e.g., Cowley, 1981a). A corresponding term to be added to the right hand side of Eq. (2) can be constructed by assuming that the twisting angle increases downtail and is linearly proportional to the IMF $B_{y}$. With these considerations in mind, the final model function $Z_{\mathrm{s}}$ was adopted in the form

$$
\begin{aligned}
Z_{\mathrm{s}} & =R_{\mathrm{H}} \tan \Psi\left\{1-\left[1+\left(\frac{\rho}{R_{\mathrm{H}}}\right)^{\alpha}\right]^{1 / \alpha}\right\} \\
& \times\left(a_{0}+a_{1} \cos \phi\right)+T \frac{B_{y}}{B_{y 0}}\left(\frac{\rho}{\rho_{0}}\right)^{\beta} \sin \phi,
\end{aligned}
$$

where the IMF $B_{y}$ and the coordinate $\rho$ in the second term were scaled for numerical convenience by $B_{y 0}=5 \mathrm{nT}$ and $\rho_{0}=10 R_{\mathrm{E}}$, respectively. The last term describes the tail twisting, quantified by the magnitude coefficient $T$ and the power index $\beta$ which controls the rate of the outward increase of the tail current rotation angle.

\section{Model parameterization by the interplanetary input variables}

In order to optimally define the final global mathematical form of the model parameters, we first fitted Eq. (3) to data subsets, corresponding to sequences of restricted intervals of $P_{\text {dyn }}$ and IMF $B_{z}$, which made it possible to at least roughly assess the impact of both factors. In general, the obtained results were found to agree well with the basic tendencies revealed earlier in TF04, namely, (1) a clear trend of the spatial scales to shrink with growing external pressure, and (2) a significantly larger elasticity of the current sheet in response to the dipole tilt and IMF $B_{y}$ during intervals of northward IMF $B_{z}$ and, conversely, stiffer geometries for southward IMF conditions. The most outstanding, stable, and robust effect revealed in the trial runs was a distinct and monotonous decrease of the hinging distance $R_{\mathrm{H}}$ with growing $P_{\mathrm{dyn}}$. It was also found that allowing $R_{\mathrm{H}}$ and $\alpha$ to vary with longitude tangibly improved the model's figure of merit, and both these parameters were found to significantly change between noon and midnight. Based on all these facts and previous experience with the TF04 model, in the final version we represented the parameters entered in Eq. (3) as simple first-order polynomials,

$$
\begin{aligned}
R_{\mathrm{H}}= & R_{\mathrm{H} 0}+R_{\mathrm{H} 1} f_{P}+R_{\mathrm{H} 2} f_{B_{z}}+ \\
& \left(R_{\mathrm{H} 3}+R_{\mathrm{H} 4} f_{P}+R_{\mathrm{H} 5} f_{B_{z}}\right) \cos \phi, \\
T= & T_{0}+T_{1} f_{P}, \\
a_{0}= & a_{00}+a_{01} f_{P}+a_{02} f_{B_{z}}, \\
a_{1}= & a_{10}+a_{11} f_{P}+a_{12} f_{B_{z}}, \\
\alpha= & \alpha_{0}+\alpha_{1} \cos \phi+\alpha_{2} f_{P}+\alpha_{3} f_{B_{z}}, \\
\beta= & \beta_{0}+\beta_{1} f_{B_{z}},
\end{aligned}
$$

of the controlling variables:

$$
f_{P}=\left(\frac{P_{\mathrm{dyn}}}{\left\langle P_{\mathrm{dyn}}\right\rangle}\right)^{\chi}-1 \quad \text { and } \quad f_{B_{z}}=\frac{B_{z}}{B_{z 0}},
$$


where the pressure $P_{\mathrm{dyn}}$ and IMF $B_{z}$ were normalized for convenience by their standard nominal values $\left\langle P_{\text {dyn }}\right\rangle=$ $2.0 \mathrm{nPa}$ and $B_{z 0}=5 \mathrm{nT}$, respectively. It should be noted here that, while the pressure values in Eq. (10) were 5 min averages, strictly corresponding to the $B_{\mathrm{r}}$ reversal times, the IMF $B_{y}$ and $B_{z}$ values were averaged over $35 \mathrm{~min}$ intervals immediately preceding the reversals, to take into account the finite response time of the system due to propagation and/or magnetic flux accumulation effects.

In total, the model included 21 free parameters, determined by minimizing the mean distance between the model surface Eqs. (3)-(10) and 109363 points in the abovedescribed cumulative crossings set. Initial starting values of the unknown parameters were set using their estimates, obtained from many preliminary trial runs with simpler and less flexible models based on a fewer number of terms. The distances between the model surface and individual data points were calculated using an iterative search algorithm performing a rapidly converging sequence of steps (Newton's method): $\boldsymbol{r}_{i+1}=\boldsymbol{r}_{i}+\Delta \boldsymbol{s}_{i}$, where the step size (approximately in the direction normal to the surface) equals $\Delta s=-\Phi \nabla \Phi /|\nabla \Phi|^{2}$ and $\Phi=z-Z_{\mathrm{s}}$.

To improve the fitting robustness, two modifications were made to the standard procedure. First, instead of using the standard optimization criterion based on the root mean square (rms) deviation of the model from data, we used the mean absolute deviation (Press et al., 1992, p. 605), such that the target function to minimize was

$Q=\frac{1}{N} \sum_{j=1}^{N} \Delta S_{j}=\frac{1}{N} \sum_{j=1}^{N}\left|\boldsymbol{r}_{j, \text { data }}-\boldsymbol{r}_{j, \text { model }}\right|$.

Second, to reduce the effect of outlier data points, the fitting was done in two stages. At the first stage, the minimization of $Q$ was made using all the data, after which data points with $\Delta S_{j}>6 Q$ were tagged with a rejection flag. At the second stage, the next fitting cycle was performed, but now without the data records previously marked as rejected. The assumed $6 Q$ threshold resulted in only a minor reduction of the original data set size (by $0.82 \%$ ), but yielded a tangible $(5.7 \%)$ improvement of the fitting figure of merit (from $Q=0.557 R_{\mathrm{E}}$ down to $\left.0.525 R_{\mathrm{E}}\right)$.

The fitting algorithm used in the derivation of the model parameters was a version of the Nelder-Mead simplex method (Press et al., 1992, p. 402). In spite of a relatively large number of nonlinear parameters, it provided sufficiently stable convergence to optimal values, mostly due to a careful choice of their starting estimates.

A standard way to characterize the quality of a model is to compare the residual (minimum) value of the target function with the average value of the modeled quantity. For example, when constructing a model of a vector field $\boldsymbol{B}$, we estimate the model's merit by the ratio of the residual rms deviation of the model field from an experimental data sample, $\Delta B=\left\langle\left|\boldsymbol{B}_{\text {data }}-\boldsymbol{B}_{\text {model }}\right|\right\rangle$, to the average magnitude $\left\langle\left|\boldsymbol{B}_{\text {data }}\right|\right\rangle$
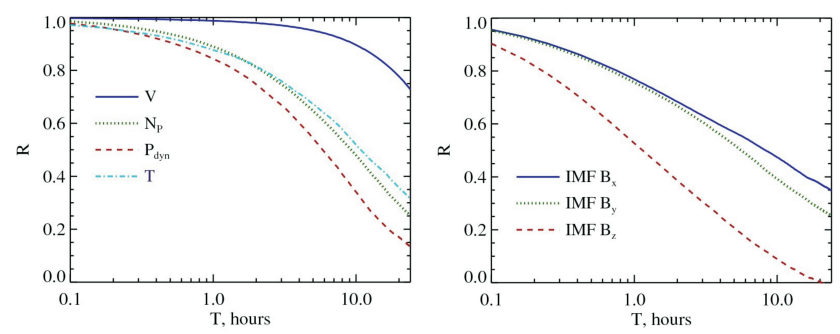

Figure 4. Autocorrelation coefficients for four principal characteristics of the solar wind (left) and three IMF components (right) as functions of the time lag $T$ in hours. The plots are based on $5 \mathrm{~min}$ average OMNI data in 19 yearly files (1995-2013).

of the observed field. The latter quantity is equal to $\Delta B$, calculated under assumption $\boldsymbol{B}_{\text {model }}=0$, i.e., without any information about the modeled field. By analogy, in the present model the assumption of no information at all about the tiltand IMF $B_{y}$-related deformation of the current is equivalent to reducing the warped sheet to the planar surface $Z_{\mathrm{GSM}}=0$. Therefore, we define the average value of the modeled quantity as the mean absolute deviation $\langle D\rangle=1.73 R_{\mathrm{E}}$ of the data points from the plane $Z_{\mathrm{SM}}=0$ and compare it with their residual deviation $Q=0.525 R_{\mathrm{E}}$ from the best-fit model surface of $B_{\mathrm{r}}$ reversals defined by Eq. (3). Based on these values, the figure of merit $Q /\langle D\rangle=0.30$, which is fairly typical for models of this kind, derived from noisy sets of spacecraft data.

In this regard, it is also interesting to compare the performances of the present TAG14 model and the earlier TF04 model in terms of the mean absolute deviation $Q$, using the same data. Since the TF04 model was derived from only nightside observations, its validity region is limited to locations with negative $X_{\mathrm{GSW}}$. For that reason, we compared the values of $Q$ using a smaller subset of the entire crossings data set, comprised of 66506 records with $X_{\mathrm{GSW}}<0$. Calculation for the TAG14 and TF04 models yielded, respectively, $Q_{\mathrm{TAG} 14}=0.63 R_{\mathrm{E}}$ and $Q_{\mathrm{TF} 04}=0.67 R_{\mathrm{E}}$. Although the improvement is not large, it should be remembered that our primary goal was not to just increase the accuracy, but to extend the model's validity region to all local times.

\section{Model fitting results}

Table 1 presents the final result of fitting the model surface Eqs. (3)-(10) to the crossings data set. Before proceeding to the specific parameters, a comment on the calculation of their uncertainties is in order. As in TF04, we used a version of the bootstrap method (Press et al., 1992, p. 686), based on 40 synthetic subsets generated from the original data. However, using the entire crossings data set for that purpose would result in significantly underestimated uncertainty values. The reason is that consecutive records corresponding to sequential observations along spacecraft orbits are sepa- 
Table 1. The model parameter values.

\begin{tabular}{cccccc}
\hline Parameter & Value & Parameter & Value & Parameter & Value \\
\hline$R_{\mathrm{H} 0}$ & $11.02 \pm 0.05$ & $T_{1}$ & $0.18 \pm 0.08$ & $\alpha_{0}$ & $7.13 \pm 0.06$ \\
$R_{\mathrm{H} 1}$ & $6.05 \pm 0.88$ & $a_{00}$ & $2.91 \pm 0.02$ & $\alpha_{1}$ & $4.87 \pm 0.07$ \\
$R_{\mathrm{H} 2}$ & $0.84 \pm 0.09$ & $a_{01}$ & $-0.16 \pm 0.07$ & $\alpha_{2}$ & $-0.22 \pm 0.12$ \\
$R_{\mathrm{H} 3}$ & $-2.28 \pm 0.08$ & $a_{02}$ & $0.56 \pm 0.03$ & $\alpha_{3}$ & $-0.14 \pm 0.04$ \\
$R_{\mathrm{H} 4}$ & $-0.25 \pm 0.37$ & $a_{10}$ & $1.89 \pm 0.03$ & $\chi$ & $-0.29 \pm 0.03$ \\
$R_{\mathrm{H} 5}$ & $-0.96 \pm 0.16$ & $a_{11}$ & $0.06 \pm 0.04$ & $\beta_{0}$ & $2.18 \pm 0.09$ \\
$T_{0}$ & $0.29 \pm 0.02$ & $a_{12}$ & $0.49 \pm 0.04$ & $\beta_{1}$ & $0.40 \pm 0.11$ \\
\hline
\end{tabular}
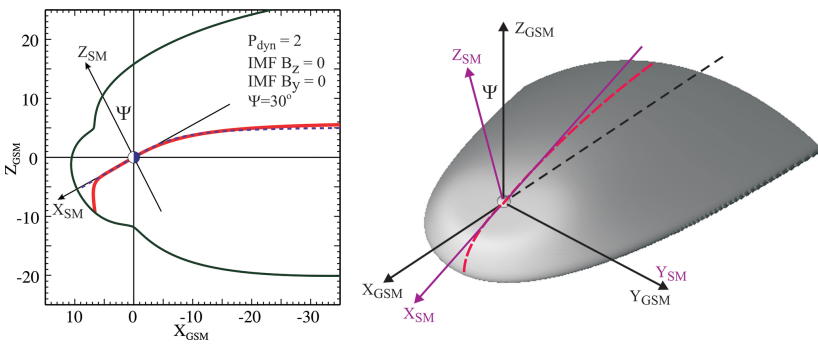

Figure 5. Left: noon-midnight section of the model surface $B_{\mathrm{r}}=0$ for average interplanetary conditions and dipole tilt angle $\Psi=30^{\circ}$ (heavy red contour). In this and following figures, the blue dashed line shows the result of the TF04 model, obtained for the same values of input parameters. The model magnetopause by Lin et al. (2010) is also shown with a magenta solid line. Right: a 3-D view of the deformed bowl-shaped model surface for the same input parameters.

rated by only 5 min intervals and, hence, cannot be treated as statistically independent because of a finite autocorrelation time of the incoming solar wind parameters (e.g., Petrinec and Russell, 1996) and owing to relatively slow variation of the dipole tilt angle. To better illustrate this point, we plotted in Fig. 4 (left panel) the autocorrelation coefficients for four principal characteristics of the solar wind (speed, proton density, temperature, and ram pressure) and for three components of the IMF (right panel), based on 19 yearly OMNI data with 5 min resolution. The ram pressure autocorrelation falls down to $R=0.6$ no sooner than in $T=4 \mathrm{~h}$, while the coherence time for IMF $B_{z}$ is much shorter, so that by $T=4$ its autocorrelation drops below 0.2 . With these results in mind, a subsampling procedure was applied to the original crossings data set, such that each record in the decimated subsets was separated from its nearest neighbors by at least $3 \mathrm{~h}$ or even by a longer interval (in case of large data gaps). Because of that restriction, the size of each synthetic subset was much smaller, being only approx. one-sixth of the original crossings set. However, the corresponding best-fit parameters did not depart too far from those obtained using the full set, and their rms deviations were adopted as the uncertainty values given in Table 1.
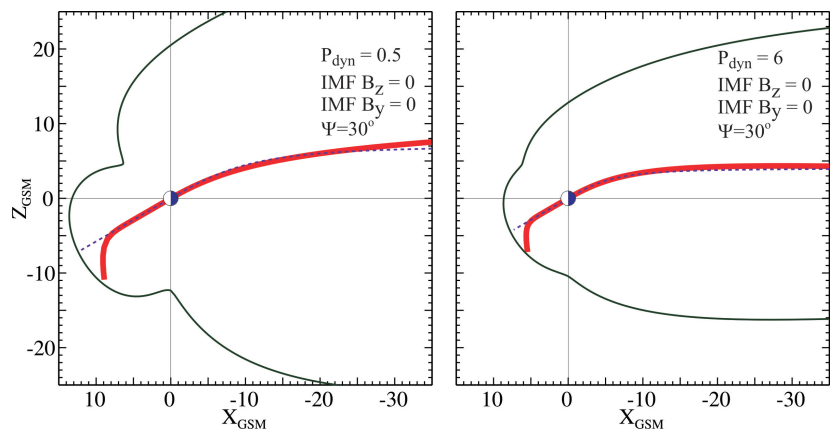

Figure 6. Illustrating the effect of the solar wind $P_{\mathrm{dyn}}$ on the size and shape of the model surface $B_{\mathrm{r}}=0$. In both cases, IMF $B_{y}=$ $B_{z}=0$.

The first five quantities in Table 1 define the hinging distance $R_{\mathrm{H}}$ as a function of longitude and interplanetary parameters. Under average conditions with $P_{\mathrm{d}}=2 \mathrm{nPa}$ and IMF $B_{z}=0, f_{P}=f_{B_{z}}=0$, the hinging distance equals $13.3 R_{\mathrm{E}}$ at midnight, but falls down to $8.75 R_{\mathrm{E}}$ in the noon sector. The $\alpha$ parameter shows even more dramatic longitude dependence, being equal to $\alpha=12$ at noon, but dropping down to $\alpha=2.3$ at midnight. The parameters $a_{0}$ and $a_{1}$ which control the bending magnitude and its day-night asymmetry, only weakly depend on the ram pressure, but are remarkably sensitive to the IMF $B_{z}$. These three effects combined result in a very gradual and smooth tilt-related bending of the surface in the night sector, contrasted by its sharp kink-like deflection from the dipole equator on the dayside, as shown in Fig. 5 (left panel), displaying the meridional section of the deformed sheet for average interplanetary conditions with $P_{\text {dyn }}=2 \mathrm{nPa}$ and IMF $B_{z}=0$ (red solid line). For comparison, the shape of the TF04 sheet for the same interplanetary conditions is shown by a blue dashed line (extrapolated on the dayside by straight line in the dipole equatorial plane). A model magnetopause by Lin et al. (2010) for the same set of input parameters is also shown with green solid line. The right panel displays a 3-D view of the model surface for the same set of input parameters.

In agreement with earlier results (TF04), a clear dependence of $R_{\mathrm{H}}$ on the ram pressure $P_{\mathrm{dyn}}$ was found: at the low end of the range, $P_{\mathrm{dyn}}=0.5 \mathrm{nPa}$, the hinging distance in- 

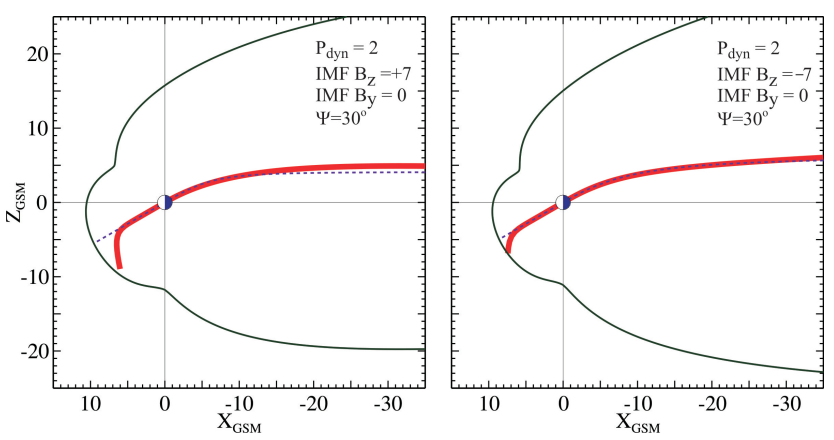

Figure 7. Illustrating the effect of the IMF $B_{z}$ on the global shape of the model surface $B_{\mathrm{r}}=0$. In both cases $P_{\mathrm{dyn}}=2 \mathrm{nPa}$.
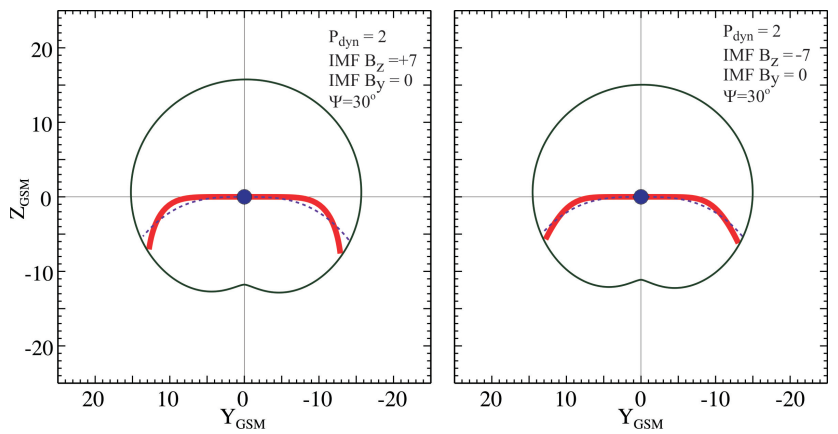

Figure 8. Illustrating the effect of the IMF $B_{z}$ on the shape of the $B_{\mathrm{r}}=0$ surface in the terminator plane. In both cases $P_{\mathrm{dyn}}=2 \mathrm{nPa}$.

creases by $3 R_{\mathrm{E}}$ from its average value, while at $P_{\mathrm{d}}=5 \mathrm{nPa}$ it decreases by $1.4 R_{\mathrm{E}}$. As for the IMF $B_{z}$ impact on $R_{\mathrm{H}}$, it is very small at noon due to near cancelation of terms with $f_{B_{z}}$ in Eq. (4), but becomes quite tangible at midnight, where $R_{\mathrm{H}}$ increases by $3.6 R_{\mathrm{E}}$ as the IMF $B_{z}$ changes from -5 to $+5 \mathrm{nT}$. Note however that, with regard to the resultant tiltrelated deformation, variations of $R_{\mathrm{H}}$ in response to the interplanetary parameters are largely interdependent with concurrent changes in the coefficients $a_{0}$ and $a_{1}$ in Eqs. (3), (6), and (7), which results in rather large errors in the obtained values of $R_{\mathrm{H} 1}, R_{\mathrm{H} 4}, R_{\mathrm{H} 5}$, and $\alpha_{2}$. As for the twisting parameter $T_{1}$, its relatively large error is due to generally noisier data in the distant tail, where the IMF $B_{y}$ effect becomes tangible. The most important net effects of $P_{\mathrm{dyn}}$ and IMF $B_{z}$ variations are illustrated in the following sequence of plots in Figs. 6-9.

Figure 6 compares the shapes of the equatorial sheet for small and large values of the solar wind ram pressure. The corresponding inflation/compression of the magnetosphere results in expansion/contraction of the sphere of influence of the geodipole, so that stronger/weaker pressures cause the sheet to depart from the SM equator at closer/larger distances.

In the next plots, Figs. 7-9, the effect of the IMF is demonstrated for two opposite extreme cases of large positive and large negative values, $B_{z}=+7 \mathrm{nT}$ and $B_{z}=-7 \mathrm{nT}$. As was
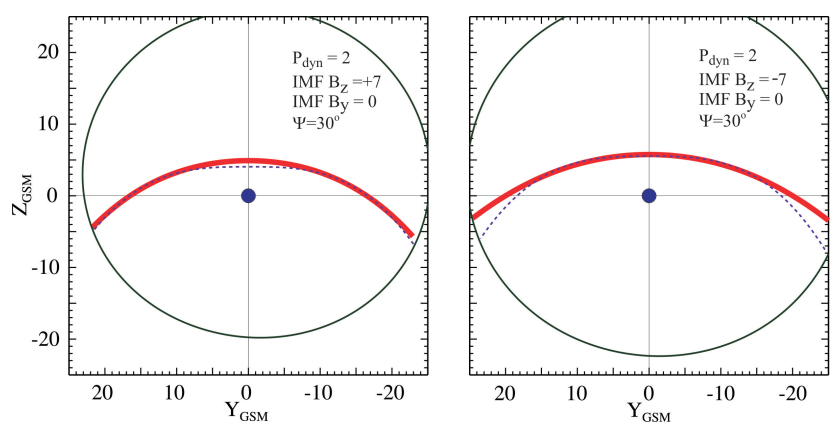

Figure 9. Illustrating the effect of the IMF $B_{z}$ on the shape of the current sheet in the distant tail cross section at $X_{\mathrm{GSW}}=-30 R_{\mathrm{E}}$. In both cases $P_{\text {dyn }}=2 \mathrm{nPa}$.
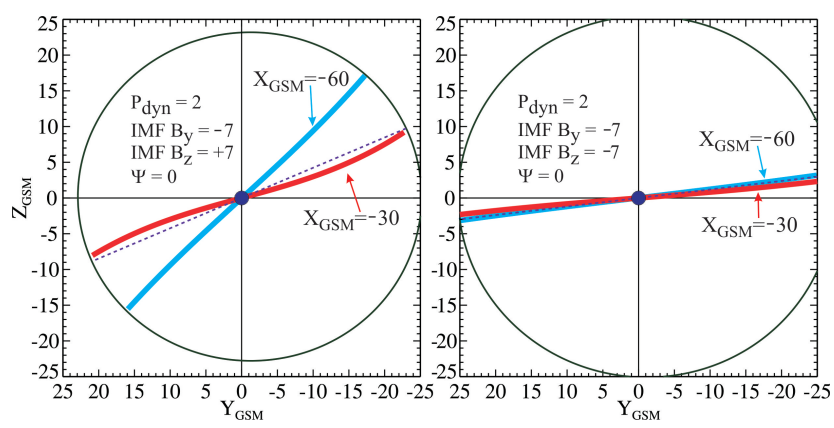

Figure 10. Effect of the IMF $B_{z}$ on the magnitude of the current sheet twisting in the distant tail cross section at $X_{\mathrm{GSW}}=-30 R_{\mathrm{E}}$. In both cases $P_{\mathrm{dyn}}=2 \mathrm{nPa}$ and IMF $B_{y}=-7 \mathrm{nT}$. Radial variation of the twisting magnitude is illustrated by the blue line, corresponding to a more distant cross section at $X_{\mathrm{GSW}}=-60 R_{\mathrm{E}}$. Note the dramatic difference in the rotation angles between the cases of northward (left) and southward (right) IMF.

found earlier in TF04, the IMF $B_{z}$ polarity affects the degree of the current sheet rigidity in response to both dipole tilt and IMF $B_{y}$, in the sense that negative $B_{z}$ results in larger values of the hinging distance $R_{\mathrm{H}}$ and weaker twisting, while positive $B_{z}$ has the opposite effect by making the tail more elastic and flexible. The present study fully confirms that finding, as can be seen in Figs. 7-9. In general, the new model agrees quite well with TF04 on the nightside, except for a somewhat more pronounced deformation in the dawn-dusk sector (Fig. 8) and a less deformed sheet at $X=-30 R_{\mathrm{E}}$ for negative IMF $B_{z}$ (Fig. 9, right panel).

Figure 10 illustrates the twisting of the current sheet in the distant tail, caused by the negative azimuthal IMF $B_{y}=$ $-7 \mathrm{nT}$ in the case of positive (left) and negative (right) IMF $B_{z}$. As already said, the magnitude of the effect is much smaller for southward IMF conditions. Note that the apparent asymmetry in the magnetopause location is due to a small residual shift present in the Lin et al. (2010) model boundary. 


\section{Discussion}

As already noted, the above-described model was derived from a set of data taken in the low-latitude magnetosphere, corresponding to single and multiple reversals of the radial component of the observed magnetic field. Based on close association of the field reversal regions with minima of the field $B$ and maxima of the current density $j$, we term the obtained model surface as global current sheet, even though in the terrestrial magnetosphere (unlike in the case of Saturn and Jupiter) the actual transverse distribution of $j$ can largely vary in local time, being relatively thin in the tail but expanding in latitude towards the dayside as a part of the ring current. In our approach, $B_{\mathrm{r}}$ reversals are rather treated as a proxy indicator of crossing the surface of $\min \{B\}$, usually co-located with regions of the largest electric current and plasma pressure. This approach can be further substantiated by noting that in closed quasi-dipole-like configurations a reversal of $B_{\mathrm{r}}$ usually corresponds to the most distant location on a given $L$ shell, i.e., to the field line apex. We therefore can test our data selection criterion by plotting a model distribution of $\delta=R / R_{\max }$, where $R$ is the geocentric distance of a point and $R_{\max }$ is the distance to the apex of a field line, passing through that point. Figure 11 (left panel) shows a result of such a calculation in the noon-midnight plane using a simple model of a curl-free magnetic field of a tilted dipole $\left(\Psi=35^{\circ}\right)$, confined inside the T96 magnetopause (to sharpen the image, we plotted here $\delta^{3}$ instead of $\delta$ ). For comparison, the right panel shows a noon-midnight distribution in the same model of the inverse magnetic mirror ratio $\varepsilon=B_{\min } / B$, where $B$ is the local field magnitude at a given location and $B_{\min }$ is a minimal value of $B$ on the field line passing through that same location (TA14). The two distributions are remarkably similar to each other except in the immediate vicinity of the magnetopause. Based on a general fact that plasma and electric currents tend to concentrate in the regions of minimal $B$ field, we expect that the obtained sets of isointensity contours of both $\varepsilon$ and $\delta$ in the vacuum field model indicate fairly well the actual shape of deformed magnetospheric currents in a realistic configuration.

The distributions in Fig. 11 are not only quite similar to each other but also strongly resemble the obtained model shapes of the $B_{\mathrm{r}}$ reversal surface in Figs. 5-7, which justifies our choice of $B_{\mathrm{r}}$ as a suitable parameter to pinpoint the shape of the deformed $\min \{B\}$ regions. As discussed earlier in TA14, the seasonal/diurnal warping of the magnetospheric equatorial current results from the combined effect of two basic asymmetries, which are the day-night asymmetry due to the solar wind flow and the transverse north-south asymmetry due to the dipole tilt. As vividly demonstrated in the above plots, this fundamental effect shows up even in a vacuum configuration with a shielded dipole and no plasma inside the magnetosphere, which guarantees that these results are not affected by any a priori assumption on the equatorial current geometry.
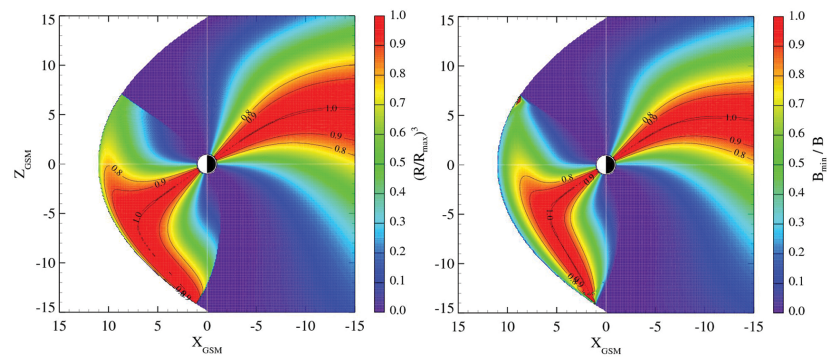

Figure 11. Illustrating the relation between the magnetic field depressions and $B_{\mathrm{r}}$ reversal areas. Left panel: distribution of $\delta=$ $R / R_{\max }$ (see text for explanation). Right panel: distribution of the inverse mirror ratio $\varepsilon=B_{\min } / B$. Both diagrams were obtained for a vacuum magnetic configuration with a tilted dipole field, shielded within a T96 model magnetopause, and correspond to the noonmidnight meridian plane $\left(Y_{\mathrm{GSM}}=0\right)$.

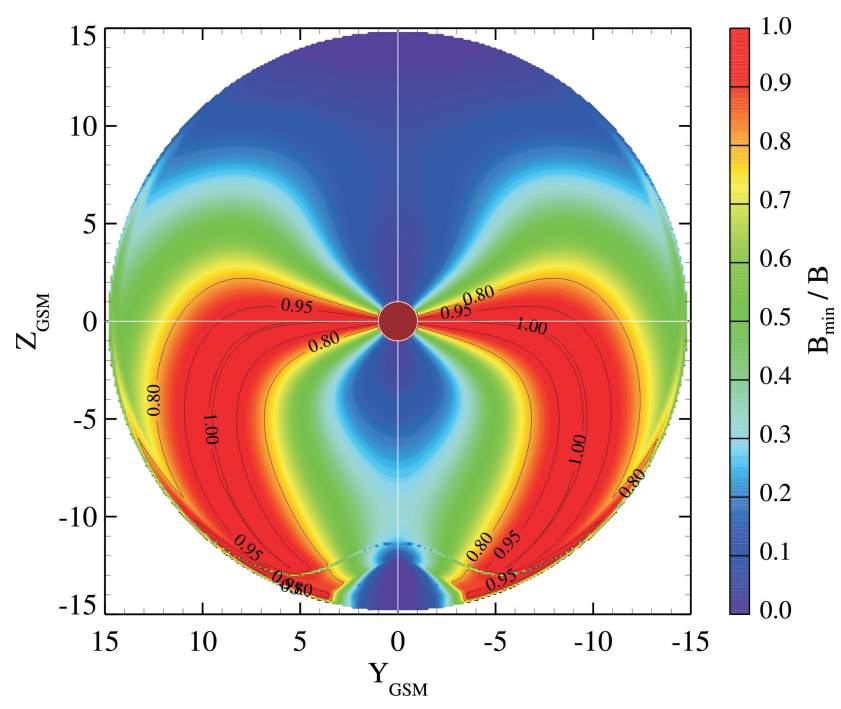

Figure 12. Distribution of the inverse mirror ratio $\varepsilon$ in a vacuum magnetic configuration with a tilted dipole field, shielded within a T96 model magnetopause. The diagram corresponds to the terminator plane $\left(X_{\mathrm{GSM}}=0\right)$.

In the next Fig. 12 a distribution of $\varepsilon$, obtained using the same procedure and the vacuum field model, but plotted in the terminator plane as a function of $\{Y, Z\}$. As can be seen from the diagram, the contours of constant $\varepsilon$ sharply bend southward at distances $R \sim 5-10 R_{\mathrm{E}}$, in a similar way to what one sees in the plots in Fig. 8.

As a future study, it would be of interest to construct a model based on independent particle data, taken during spacecraft crossings of equatorial regions with high-plasma beta, and thus obtain more information on the spatial structure of the equatorial current. Another interesting issue is a possible influence of IMF $B_{x}$ component on the position of the plasma sheet with respect to the GSM equatorial plane. The effect was theoretically predicted by Cowley (1981b) and it has indeed been observed in MHD simulations, as 


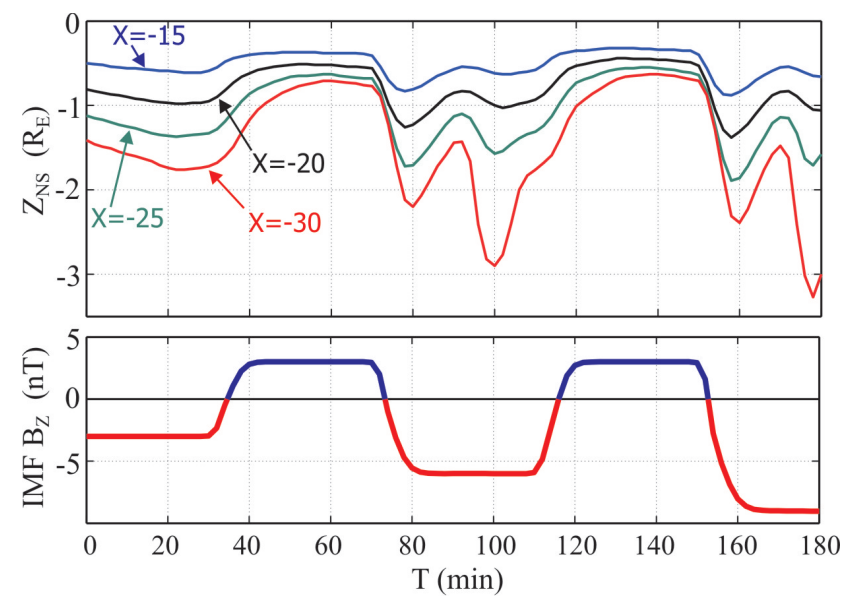

Figure 13. Time variation of the neutral sheet position $Z_{\mathrm{NS}}$ at four tailward distances (top panel), in a MHD simulation run (BATSRUS at the Coordinated Community Modeling Center (CCMC)) with a constant IMF $B_{x}=6 \mathrm{nT}$ and varying IMF $B_{z}$ (bottom panel). Southward excursions of $Z_{\mathrm{NS}}$ (up to $-3 R_{\mathrm{E}}$ at $X=-30 R_{\mathrm{E}}$ ) occur during the periods with IMF $B_{z}<0$.

shown in Fig. 13, displaying a result of an MHD simulation run with zero dipole tilt angle, in which IMF $B_{x}$ was kept constant and positive at $+6 \mathrm{nT}$, while IMF $B_{z}$ was set to periodically change its polarity in a stepwise manner. Variations of the neutral sheet deflection $Z_{\mathrm{NS}}$ from the equatorial plane at $Y=0$ are shown by different colors for four locations between $X=-15 R_{\mathrm{E}}$ and $X=-30 R_{\mathrm{E}}$. The shift magnitude increases with growing tailward distance and reaches $\sim-3 R_{\mathrm{E}}$ during intervals of southward IMF $B_{z}$.

Somewhat surprisingly, this effect has evaded detection in our data-based study. To that end, we modified the empirical model by adding a north-south shift term in Eq. (3), proportional to IMF $B_{x}$ and including various factors to account for the magnifying effect of the IMF $B_{z}$. The magnitudes of the $B_{x}$-related shift obtained from fitting the modified model to the crossings data set were found to be much smaller (within $0.1-0.2 R_{\mathrm{E}}$ ) than in MHD simulations. The source of the disagreement remains yet unclear, and it is planned to further explore this issue in a future study.

\section{Conclusions}

A new quantitative model is developed of the shape of the magnetospheric equatorial current sheet as a function of the geodipole tilt angle and the interplanetary parameters, including the solar wind ram pressure and transverse components of the IMF, averaged over preceding half-hour intervals. The current sheet is defined here as a smooth surface, optimally fitted to a set of locations where the observed magnetic field reverses its radial component. This work generalizes the model of Tsyganenko and Fairfield (2004) developed earlier for the nightside part of the current sheet, by ex- tending it to the full range of local time, including the noon sector. Fitting the model equations to observations reveals the bowl-like shape of the deformed sheet, i.e., its global deflection from the solar-magnetic equatorial plane in the antisunward direction. The effect is analogous to that reported to exist at Saturn (Arridge et al., 2008), and its presence in the Earth's magnetosphere was demonstrated and interpreted recently in Tsyganenko and Andreeva (2014). The tilt-related warping of the current on the dayside is much sharper, and its bending angle is larger than in the nightside sector, which is manifested in the strong azimuthal variation of the sharpness parameter $\alpha$ and of the hinging distance $R_{\mathrm{H}}$. The hinging distance is also quite sensitive to the ram pressure of the solar wind, so that the bowl diameter (hence, the magnitude of the sheet north-south excursions) decreases with growing $P_{\mathrm{dyn}}$. In agreement with the earlier results (TF04), the $R_{\mathrm{H}}$ parameter increases (decreases) during southward (northward) IMF periods, which results in expansion (contraction) of the dipole-dominated region, in terms of the tilt-related deformation. In other words, the current sheet becomes stiffer when IMF $B_{z}<0$ and, conversely, more elastic when IMF $B_{z}>0$. The same effect is clearly observed in the IMF $B_{y}$-related twisting of the tail current. With regard to the influence of the IMF $B_{x}$ on the tail current position, no clear effect was found, in apparent disagreement with the results of MHD simulations. We relegate a more detailed study of that issue for a future work.

Acknowledgements. It is a pleasure to acknowledge the teams and principal investigators of all the experiments whose data were used in this study. Geotail MGF data were provided by the principal investigators, S. Kokubun (STEL) and T. Nagai (Tokyo Institute of Technology, Japan). The data of Polar MGF experiment were made available online by the UCLA team led by the principal investigator C. T. Russell. NASA contract NAS5-02099 and V. Angelopoulos are acknowledged for use of THEMIS data, specifically, K. H. Glassmeier, U. Auster, and W. Baumjohann are acknowledged for the use of FGM data provided under the lead of the Technical University of Braunschweig and with financial support through the German Ministry for Economy and Technology and the German Center for Aviation and Space (DLR) under contract 50 OC 0302. The Cluster magnetometer and spacecraft ephemeris data were obtained from the NSSDC CDAWEB online facility, originally provided by the PIs, A. Balogh and M. Tatrallyay. High-resolution OMNI interplanetary data were obtained from the SPDF OMNIWEB interface (R. McGuire, N. Papitashvili). We acknowledge the CCMC facility at the GSFC for providing BATSRUS MHD simulation runs.

This work was supported by the Russian Science Foundation grant 14-17-00072.

Topical Editor C. Owen thanks T. I. Pulkkinen and one anonymous referee for their help in evaluating this paper. 


\section{References}

Angelopoulos, V.: The THEMIS mission, Space Sci. Rev., 141, 534, 2008.

Arridge, C. S., Khurana, K. K., Russell, C. T., Southwood, D. J., Achilleos, N., Dougherty, M. K., Coates, A. J., and Leinweber, H. K.: Warping of Saturn's magnetospheric and magnetotail current sheets, J. Geophys. Res., 113, A08217, doi:10.1029/2007JA012963, 2008.

Auster, H. U., Glassmeier, K. H., Magnes, W., Aydogar, O., Baumjohann, W., Constantinescu, D., Fischer, D., Fornacon, K. H., Georgescu, E., Harvey, P., Hillenmaier, O., Kroth, R., Ludlam, M., Narita, Y., Nakamura, R., Okrafka, K., Plaschke, F., Richter, I., Schwarzl, H., Stoll, B., Valavanoglou, A., and Wiedemann, M.: The THEMIS fluxgate magnetometer, Space Sci. Rev., 141, 235-264, 2008.

Balogh, A., Dunlop, M. W., Cowley, S. W. H., Southwood, D. J., Thomlinson, J. G., Glassmeier, K. H., Musmann, G., Luehr, H., Buchert, S., Acuna, M. H., Fairfield, D. H., Slavin, J. A., Riedler, W., Schwingenschuh, K., and Kivelson, M. G.: The Cluster magnetic field investigation, Space Sci. Rev., 79, 65-92, 1997.

Cowley, S. W. H.: Magnetospheric asymmetries associated with the Y-component of the IMF, Planet. Space Sci., 29, 79-96, 1981a.

Cowley, S. W. H.: Asymmetry effects associated with the Xcomponent of the IMF in a magnetically open magnetosphere, Planet. Space Sci., 29, 809-818, 1981b.

Hones, E. W., Zwickl, R. D., Fritz, T. A., and Bame, S. J.: Structural and dynamical aspects of the distant magnetotail determined from ISEE-3 plasma measurements, Planet. Space Sci., 34, 889-901, 1986.

King, J. H. and Papitashvili, N. E.: Solar wind spatial scales in and comparisons of hourly Wind and ACE plasma and magnetic field data, J. Geophys. Res., 110, A02104, doi:10.1029/2004JA010649, 2005.

Kokubun, S., Yamamoto, T., Acuna, M. H., Hayashi, K., Shiokawa, K., and Kawano, H.: The Geotail magnetic field experiment, J. Geomagn. Geoelectr., 46, 7-21, 1994.
Lin, R. L., Zhang, X. X., Liu, S. Q., Wang, Y. L., and Gong, J. C.: A three-dimensional asymmetric magnetopause model, J. Geophys. Res., 115, A04207, doi:10.1029/2009JA014235, 2010.

Nishida, A.: The Geotail mission, Geophys. Res. Lett., 21, 28712873, doi:10.1029/94GL01223, 1994.

Petrinec, S. M. and Russell, C. T.: Near-Earth magnetotail shape and size as determined from the magnetopause flaring angle, J. Geophys. Res., 101, 137-152, 1996.

Press, W. H., Teukolsky, S. A., Vetterling, W. T., and Flannery, B. P.: Numerical Recipes in Fortran, 2nd Edn., Cambridge Univ. Press, New York, 1992.

Russell, C. T., Snare, R. C., Means, J. D., Pierce, D., Dearborn, D., Larson, M., Barr, G., and Le, G.: The GGS/Polar magnetic fields investigation, Space Sci. Rev., 71, 563-582, 1995.

Tsyganenko, N. A.: Modeling of twisted/warped magnetospheric configurations using the general deformation method, J. Geophys. Res., 103, 23551-23563, 1998.

Tsyganenko, N. A.: A model of the near magnetosphere with a dawn-dusk asymmetry 2. Parameterization and fitting to observations, J. Geophys. Res., 107, SMP10.1-SMP10.17, doi:10.1029/2001JA000220, 2002.

Tsyganenko, N. A. and Andreeva, V. A.: On the "bowl-shaped" deformation of planetary equatorial current sheets, Geophys. Res. Lett., 41, 1079-1084, doi:10.1002/2014GL059295, 2014.

Tsyganenko, N. A. and Fairfield, D. H.: Global shape of the magnetotail current sheet as derived from Geotail and Polar data, J. Geophys. Res., 109, A03218, doi:10.1029/2003JA010062, 2004.

Tsyganenko, N. A., Karlsson, S. B. P., Kokubun, S., Yamamoto, T., Lazarus, A. J., Ogilvie, K. W., Russell, C. T., and Slavin, J. A.: Global configuration of the magnetotail current sheet as derived from Geotail, Wind, IMP 8, and ISEE 1/2 data, J. Geophys. Res., 103, 6827-6841, 1998. 